\title{
Comportement de l'austénite mère aux traitements lents au dessus de Af dans les alliages AMF à base de $\mathrm{Cu}-\mathrm{Al}-\mathrm{Ni}$
}

\author{
M. Bouabdallah ${ }^{1}$, H. Cheniti ${ }^{1}$ N. Achour ${ }^{1}$ et G. Cizeron $^{2}$ \\ 1 Laboratoire de Science et Génie des Matériaux, Département de Métallurgie, École \\ Nationale Polytechnique, 10 Avenue Hassen Badi, Alger, Algérie \\ ${ }^{2}$ Laboratoire de Structure des Matériaux Métalliques, Université de paris-Sud, bâtiment 414, \\ 91940 Orsay Cedex, France
}

\begin{abstract}
On heating at temperature upper then Af and then cooled at room temperature, the initially quenched structure of the $\mathrm{Cu}-\mathrm{Al}$ - Ni SMA changes significantly. These changes in the structure depend essentially of the heating speed and the maximal temperature reached. Consequently, the martensitic transformation temperatures rise up. In the present study, two modes of processing are applied on the alloy: a slow heating and cooling, and an isothermal treatment at $400{ }^{\circ} \mathrm{C}$ during several times and then cooled quickly to room temperature. The samples are observed by metallographic method and analyzed by XR diffraction. The mode of transformation of the parent phase $\beta_{1}$ into the equilibrium phase $\alpha+\gamma_{2}$ is also determined.
\end{abstract}

\section{INTRODUCTION}

Lors d'un réchauffage de la structure trempée d'un alliage AMF, la structure subit différentes évolutions dépendant essentiellement de la vitesse de chauffage et de la température maximale atteinte. Ces changements concernent principalement la phase mère $\beta_{1}$. En effet, dès que la température dépasse le point Af, la structure thermodynamiquement instable, subit une décomposition plus ou mois lente selon la température atteinte et la durée de traitement. Le processus s'effectue en plusieurs étapes successives [1]. Par contre, lors d'un chauffage lent et progressif jusqu'à une température du domaine monophasé $\beta$ on enregistre dans un appareil de DSC différentes évolutions notées respectivement $\mathrm{A}, \mathrm{B}, \mathrm{C}, \mathrm{D}$ et $\mathrm{E}$ (figure 1).

L'objectif de notre étude est de préciser la nature des phases formées durant un vieillissement prolongé à une température élevée (comprise entre 400 et $550^{\circ} \mathrm{C}$ ). Dans cet intervalle de température les transformations de l'austénite mère sont complexes. En effet, un chauffage relativement rapide $\left(\mathrm{Vc}>2{ }^{\circ} \mathrm{C} / \mathrm{min}\right.$ ) entraîne une réaction endothermique. Par contre un chauffage très lent provoque une réaction exothermique [2]. Un chauffage rapide entraînerait une redissolution partielle des précipités formés durant le séjour aux températures plus basses, en l'occurrence dans l'intervalle de l'évolution B. Par contre, le chauffage lent et/ou un maintien isotherme dans l'intervalle de température compris entre 400 et $550{ }^{\circ} \mathrm{C}$ conduirait à la décomposition de la phase mère donnant les phases d'équilibre. Le mode de transformation ainsi que les cinétiques font l'objet de travail. 


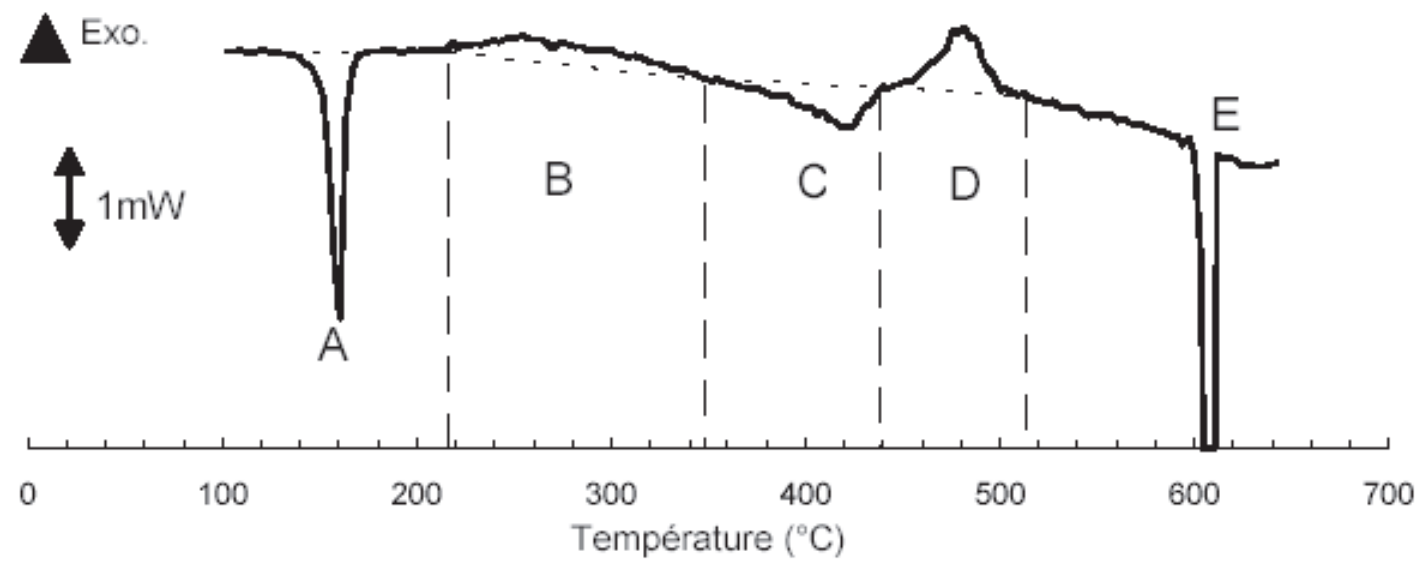

Figure 1. Exemple typique de diagramme DSC obtenu au cours d'un chauffage lent $\left(2{ }^{\circ} \mathrm{C} / \mathrm{mn}\right)$ d'un échantillon $\mathrm{AMF}$ du type $\mathrm{Cu}-\mathrm{Al}-\mathrm{Ni}$ préalablement trempé et revenu [2].

Le matériau étudié a été fourni par la société TREFIMETAUX, C'est un alliage à base de cuivre dont la composition massique nominale est de $13.3 \% \mathrm{Al}$ et $4 \% \mathrm{Ni}$, élaboré par fusion classique puis filé à chaud.

\section{INFLUENCE DE LA TEMPÉRATURE MAXIMALE ATTEINTE}

En appliquant un cycle de chauffage $\left(5^{\circ} \mathrm{C} / \mathrm{min}\right)$ à une température juste au dessus de Af suivi d'un refroidissement à la même vitesse, on enregistre une transformation réversible martensite $\leftrightarrow$ austénite avec une hystérésis d'environs $8{ }^{\circ} \mathrm{C}$. Par contre, en poussant le chauffage à une température supérieure à celle de l'évolution $\mathrm{B}$, on enregistre au refroidissement une transformation austénite $\rightarrow$ martensite «prématurée» (hystérésis négatif $-30{ }^{\circ} \mathrm{C}$ ) (figure 2). Ceci est due aux phénomènes de précipitation de phases d'équilibre riches en aluminium appauvrissant ainsi la matrice, d'où le déplacement des points de transformation vers des températures plus élevées [3]. L'analyse par DSC montre que le taux de martensite formée au refroidissement dépend également de la température maximale et de la durée de séjour à cette température. En effet, en augmentant la température maximale de traitement, au refroidissement, le taux de martensite formé diminue (figure $2(b)$ ).

Dans la figure 3 (a), est présenté un diagramme DSC au chauffage à $5{ }^{\circ} \mathrm{C} / \mathrm{min}$ jusqu'à $450{ }^{\circ} \mathrm{C}$ ensuite, $0.5^{\circ} \mathrm{C} / \mathrm{min}$ jusqu'à la température de $550^{\circ} \mathrm{C}$ suivi d'un refroidissement à $5^{\circ} \mathrm{C} / \mathrm{min}$ jusqu'à $80^{\circ} \mathrm{C}$.

$\mathrm{Au}$ cours de la deuxième phase de chauffage, on remarque nettement une réaction exothermique (figure 3 (b)). Cette réaction correspond à la décomposition complète de la phase $\beta_{1}$ en phase $\alpha$ et $\gamma_{2}$, puisque au refroidissement on n'enregistre aucune évolution. On constate que cette transformation est très lente et irréversible. L'énergie dégagée lors de cette évolution $(7.25 \mathrm{~J} / \mathrm{g})$ est voisine de celle de la transformation martensitique thermoélastique de l'alliage étudié (environ $8 \mathrm{~J} / \mathrm{g}$ ). 


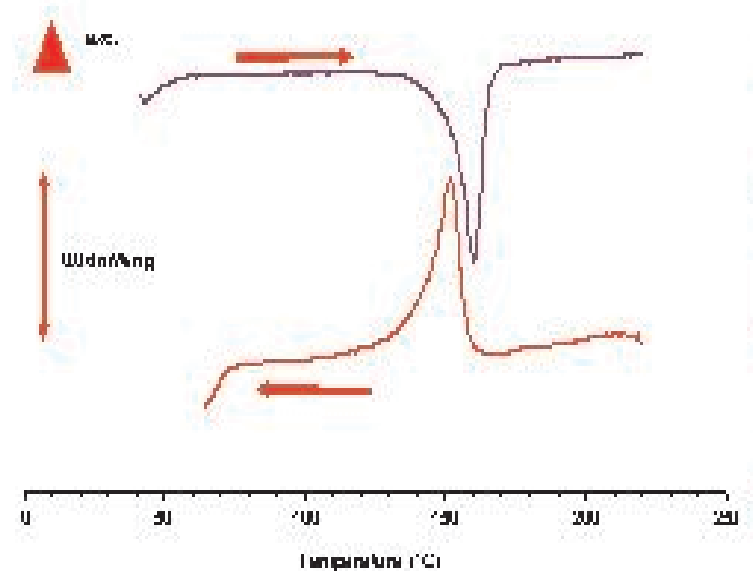

(a)

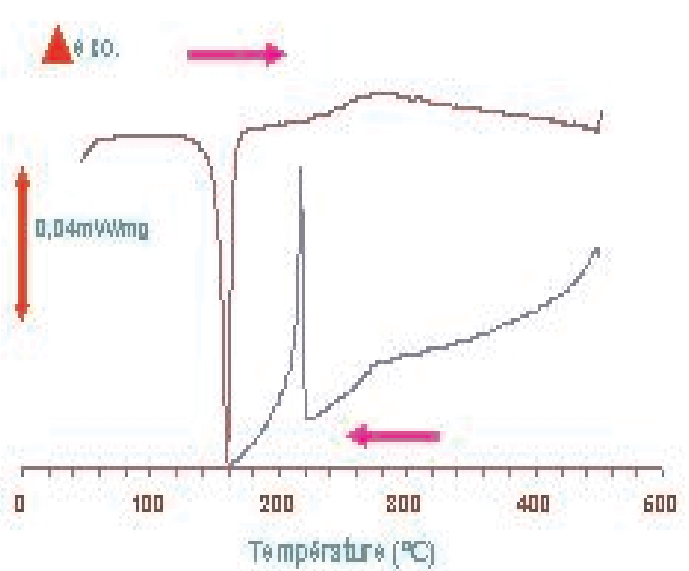

(b)

Figure 2. (a) courbes de chauffage-refroidissement à $5^{\circ} \mathrm{C} / \min \mathrm{Tmax}=220{ }^{\circ} \mathrm{C}$. (b) courbes de chauffagerefroidissement à $5^{\circ} \mathrm{C} / \min \operatorname{Tmax}=450^{\circ} \mathrm{C}$.

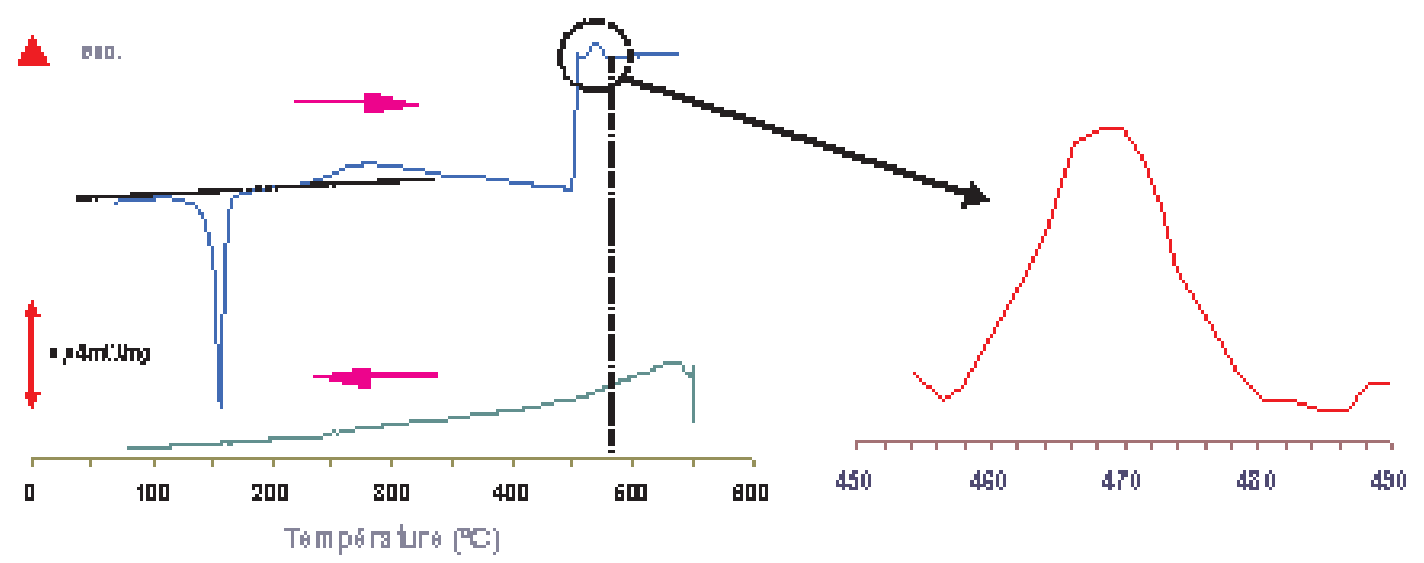

(a)

(b)

Figure 3. (a) courbe de chauffage en deux étapes (de 50 à $450{ }^{\circ} \mathrm{C} \quad 5^{\circ} \mathrm{C} / \mathrm{min}$ et de 450 à $550{ }^{\circ} \mathrm{C} \quad 0.5^{\circ} \mathrm{C} / \mathrm{min}$ ) et refroidissement à $5{ }^{\circ} \mathrm{C} / \mathrm{min}$. (b) zoom de la partie montrant la transformation exothermique entre 458 et $480{ }^{\circ} \mathrm{C}$.

\section{INFLUENCE D'UN TRAITEMENT ISOTHERME}

En appliquant un traitement isotherme au dessus du domaine de l'évolution $\mathrm{B}\left(\mathrm{T}=400{ }^{\circ} \mathrm{C}\right)$ à la structure de trempe, l'austénite mère se décompose selon un processus conduisant à la formation simultanée des phases d"équilibre $\left(\alpha+\gamma_{2}\right)$. La germination de la cellule biphasée s"effectue presque exclusivement aux joints de grains de la phase mère $\beta_{1}$ (figure 4 (a)). 


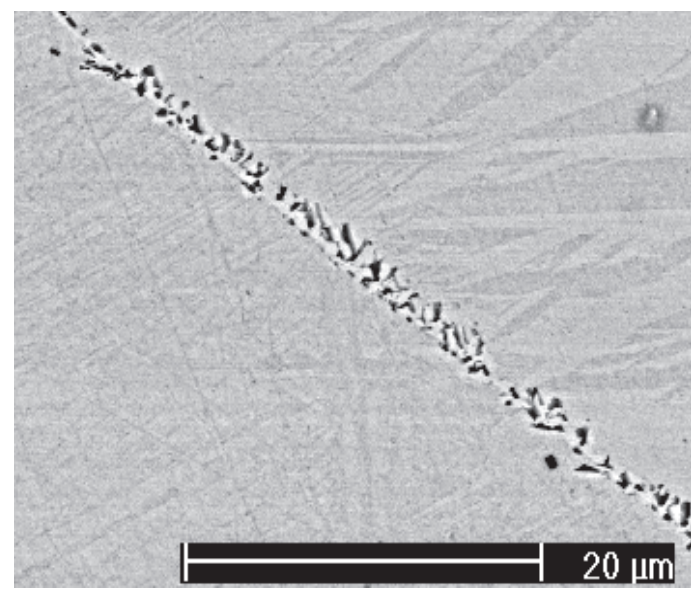

(a)

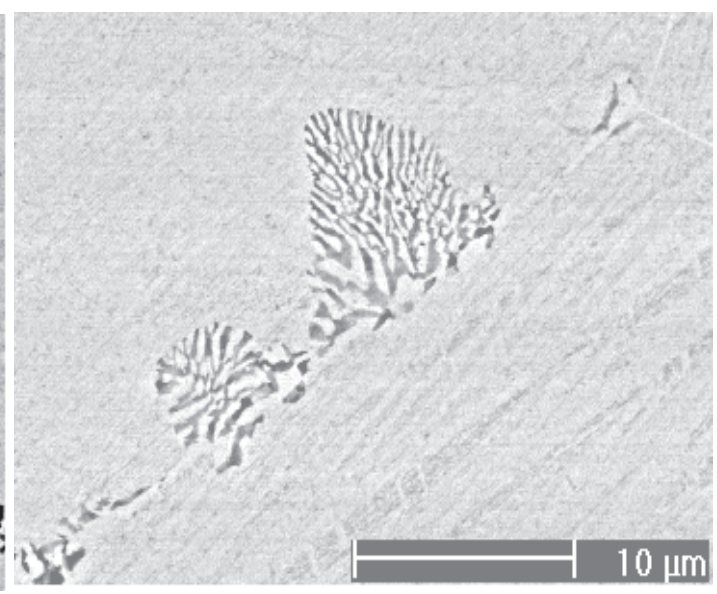

(b)

Figure 4. Micrographies électroniques obtenues à partir de la structure de trempe après vieillissement à $400{ }^{\circ} \mathrm{C}$ pendant (a) $10 \mathrm{~min}$, (b) $20 \mathrm{~min}$.

La croissance se poursuit en développant des lamelles alternées orientées vers le cœur du grain de phase mère (figure 4 (b)). Il est remarqué que pendant la formation de nouvelles ramifications de la phase $\gamma_{2}$ (en gris foncé sur les micrographies), la matrice au voisinage immédiat s'appauvrit en aluminium et se transforme en phase $\alpha$ (de couleur gris claire sur les micrographies). Simultanément, l'épaisseur des lamelles biphasées formées antérieurement augmente indiquant un phénomène de coalescence.

Initialement, la croissance d'une colonie biphasée s'effectue dans le sens longitudinal et dans le sens radial par rapport au joint de grain. La croissance longitudinale cesse lorsque les colonies se rencontrent. Ainsi, le front de réaction se trouve réduit entraînant une cinétique de croissance de plus en plus lente. De ces remarques on conclue que le mode de précipitation des cellules biphasées $\alpha+\gamma_{2}$ s'effectue par un mode continu.

L'analyse par diffraction des RX d'échantillons initialement trempés et ayant subit un vieillissement à $400^{\circ} \mathrm{C}$ durant un temps échelonné suivie d'un refroidissement à l'eau montre une évolution progressive (figure 5). La microstructure de trempe est essentiellement martensitique du type $\beta_{1}^{\prime}$. Les raies de diffraction présentes sur le diagramme de l'échantillon n'ayant pas subit un vieillissement (diagramme NT) correspondent respectivement a (00 18) $\beta_{1}^{\prime}$, (12-8) $\beta_{1}^{\prime}$ et (12 10) $\beta_{1}^{\prime}$.

Après le maintien à $400{ }^{\circ} \mathrm{C}$, la raie $(00$ 18) de la martensite disparaît progressivement en faveur de la raie (111) de la phase $\alpha$. La structure de la phase mère non encore transformée au terme d'un vieillissement à $400{ }^{\circ} \mathrm{C}$ durant un temps supérieur à 40 min donne, au refroidissement ultérieur, une structure complexe dont les raies de diffraction sont moins précises. Pour une durée relativement élevée de vieillissement $\left(320 \mathrm{~min}\right.$ à $\left.400^{\circ} \mathrm{C}\right)$, la transformation complète de l'austénite mère est alors achevée. En effet la raie (411) ; (330) $\gamma_{2}$ devient plus intense.

La durée nécessaire à la décomposition complète de la phase mère à la température de $400{ }^{\circ} \mathrm{C}$ est importante (plus de $5 \mathrm{~h}$ de maintien). Ceci suggère qu'un traitement à une température plus basse nécessite un temps de maintien plus important d'où une cinétique plus lente de transformation. De ce fait, on montre que les alliages à mémoire à base de $\mathrm{Cu}-\mathrm{Al}-\mathrm{Ni}$, présentent une grande stabilité de l'austénite mère aux températures relativement élevées. 


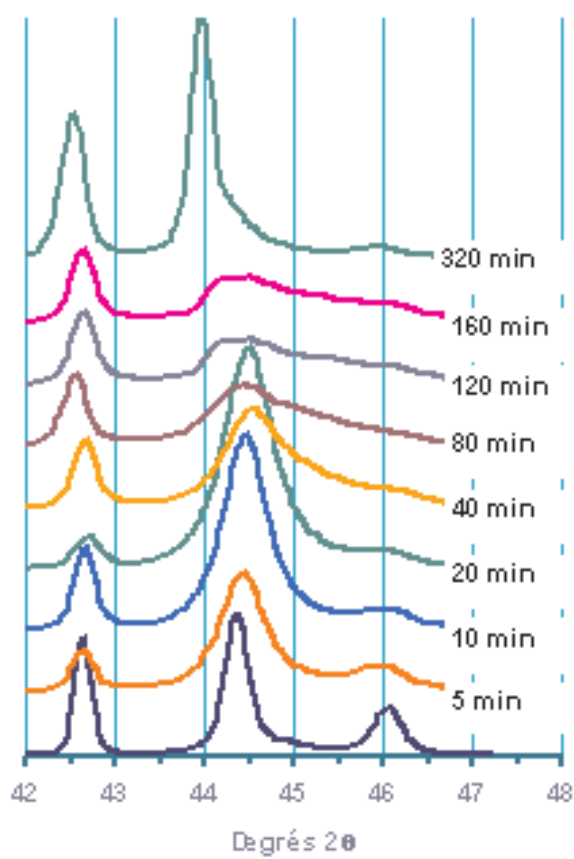

Figure 5. Diffractogrammes obtenus à partir d'échantillons initialement trempé puis vieillis à une température de $400{ }^{\circ} \mathrm{C}$ pendant une durée variable et ramenés rapidement à la température ambiante. Le diagramme NT correspond à la structure trempé et non vieillie.

\section{CONCLUSION}

Un alliage à mémoire à base de $\mathrm{Cu}-\mathrm{Al}-\mathrm{Ni}$ subit diverses transformations lorsqu'il est porté à une température relativement élevée. Les proportions de phases d'équilibre formées durant un séjour prolongé à haute température affectent irréversiblement les propriétés de thermoélasticité de la martensite. Au cours de cette étude nous montrons que :

- En augmentant la température maximale de traitement, au refroidissement, le taux de martensite formé diminue.

- Un chauffage très lent au dessus de $450{ }^{\circ} \mathrm{C}$ provoque une transformation très lente et irréversible au sein de l'austénite.

- Le mode de précipitation des cellules biphasées $\alpha+\gamma_{2}$ s'effectue par un processus continu lorsque la température maximale de traitement isotherme est fixée à $400{ }^{\circ} \mathrm{C}$.

\section{Références}

[1] M. BOUABDALLAH et G. CIZERON : Evolution of the martensitic transformation by ageing in Cu-Al- Ni SMA : Annales de Chimie Science des Matériaux., Vol. 28, 2003, pp31-39.

[2] M. BOUABDALLAH et G. CIZERON: Caractérisation par DSC des séquences de transformation au cours d'un chauffage lent des alliages $A M F$ à base de $\mathrm{Cu}-\mathrm{Al}-\mathrm{Ni}$ : Journal of Thermal Analysis and Calorimetry, Vol. 68 (3), 2002, pp. 951-956..

[3] JOGENDER SINGH, H. CHEN, AND C.M. WAYMAN: Scripta. Met., Vol. 19, 1985, p887 